\title{
Antimicrobial Resistance and Pathogenicity of Corynebacterium Striatum Clinical Isolates Collected from Three Tertiary Hospitals in China
}

\section{Junrui Wang}

Affiliated hospital of Inner mongolian medical university

\section{Xiaohong Shi}

Shandong provincial Qianfoshan Hospital

\section{Jian Zhang}

Bayannur People's hospital

\section{Xiaoli Du}

Chinese Center for Disease Control and Prevention

\section{Xuancheng Lu}

Chinese Center for Disease Control and Prevention

\section{Yuan Chai}

Affiliated hospital of Inner mongolian medical university

\section{Yingying Lv}

Affiliated hospital of Inner mongolian medical university

\section{Roushan Liu}

Affiliated Hospital of Inner Mongolia Medical University

Yanqiu Han ( $\square$ nmghyq1016@126.com )

Affiliated hospital of Inner Mongolian medical university

\section{Research}

Keywords: Corynebacterium striatum, multi-drug resistance, genotyping, Adherence, pathogenicity

Posted Date: December 10th, 2019

DOI: https://doi.org/10.21203/rs.2.18438/v1

License: (c) (i) This work is licensed under a Creative Commons Attribution 4.0 International License. Read Full License 


\section{Abstract}

Background

Antimicrobial resistance and patient-to-patient transmission of Corynebacterium striatum ( C. striatum ) clinical strains were frequently reported in recent years. Even worse, daptomycin resistant isolates were found in some studies and possible resistance mechanism was explored. Also, few investigations revealed the diversity of resistance feature and potential pathogenicity of $\mathrm{C}$. striatum strains with different genotypes. However, less is known about the possible differences of resistance feature and pathogenicity of C. straitum clinical strains from different hospitals at a long distance.

Methods

C. striatum clinical strains were isolated and identified with VITEK-2 ANC card, MALDI-TOF microTyper and 16S rRNA sequencing technique. Broth microdilution method was used to detect the antibiotic susceptibility profiles of 420 C. striatum clinical isolates, and PFGE method was used to discriminate different clones. Furthermore, in vitro adherence assay and mouse toxicity assay were performed to assess the pathogenicity of the strains with different genotypes.

Results

420 C. striatum isolates were all sensitive to vancomycin, linezolid and daptomycin. Based on antibiotic resistance results, 420 strains were classified into 19 resistance patterns, when R1, R2 and R3 patterns accounted for $45.2 \%(190 / 420), 20.2 \%(85 / 420)$ and $22.4 \%(94 / 420)$, which were all multi-drug resistant patterns. PFGE typing results showed that $107 \mathrm{C}$. striatum strains were classified into 52 types (T01-T52), when 4 epidemic clones(T36, T28, T32, T14) accounted for 14.02\% (15/107), 11.21\%(12/107), 5.61\%(6/107) and 3.73\%(4/107), respectively. All of these 4 clones belonged to resistance patterns R1, R2 and R3. Among 27 C. striatum strains, $92.6 \%(25 / 27)$ strains showed moderate to strong in vitro adherence abilities, while only $7.4 \%(2 / 27)$ strains showed weak adherence ability on polystyrene surfaces. Furthermore, mouse lethality of different strains differed greatly, when non-dominant clone(Strain NMGYC339, T24) showed the strongest mouse lethality(90.0\%).

\section{Conclusions}

The majority of C. striatum strains were multi-drug resistant and few dominant clones could persist for a long time in hospital environment. The in vitro adherence abilities and mouse lethality among different clones differed greatly. The resistance and pathogenicity of C. striatum clinical strains should be paid more attention to, especially for some specific clones at different hospitals.

\section{Introduction}

In recent years, more and more reports regarding Corynebacterium striatum (C. striatum)were published, since most of the $\mathrm{C}$. striatum strains were found to be multi-drug resistant and the only effective antibiotics were limited to vanvomycin, lizezolid [1-3]. Even worse, daptomycin resistant isolates were recently reported and its resistance mechanism was explored, which pose a more knotty issue for clinicians [4-6]. Furthermore, some recent reports revealed that $\mathrm{C}$. striatum can lead to multi-site invasive infections, such as bacteremia, 
monomicrobial bone and joint infection, prosthetic valve endocarditis [7-9], which suggest that C. striatum may possess stronger pathogenicity, especially for the patients with specific risk factors $[10,11]$.

It is reported that $\mathrm{C}$. striatum can colonize onto multiple environmental and bodily surfaces and could transmit within patients in specific wards $[12,13]$. Also, the multi-drug resistant $\mathrm{C}$. striatum clones seemed to be with stronger adhesion abilities ${ }^{[14]}$, which was consistent with one of our previous study [15], which may partly explain the reason why C. striatum can rapidly transmit within hospital environment. Moreover, Souza C et al [16] reported that $\mathrm{C}$. striatum could lead to the death of Caenorhabditis elegans, and virulence potential varied among different $\mathrm{C}$. striatum clones. The pathogenicity potential of $\mathrm{C}$. striatum strains isolated from different origins need to be further investigated.

In this study, we aim to perform a further investigation with a larger number of $\mathrm{C}$. striatum isolates from multicentres to better understand the actual resistance and pathogenicity features of C. striatum. Here, C. striatum isolates were collected in three tertiary teaching hospitals in China from March 2013 to May 2019, and the antibiotic susceptibility features were tested. Furthermore, the potential pathogenicity of different clones with different genotypes were investigated.

\section{Materials And Methods}

\section{C. striatum isolation and identification}

The hospitals enrolled in this study include Affiliated hospital of Inner Mongolian medical University (hospital A, 3000 beds), Shandong Provincial QianFuoShan Hospital(hospital B, 2813 beds) and Bayannaoer People's Hospital (hospital C, 1700 beds), China. From March 2013 to May 2019, all of C. striatum strains isolated from aseptic sites were collected and identified. The sputum samples were enrolled if they were evaluated to be qualified based on the numbers of leukocytes and epithelieum using microscopy [13]. All of the cultures suspected to be C. striatum were routinely identified with VITEK-2 ANC card(Biometrieum, France), MALDI-TOF microTyper (Tianrui, China) and 16S rRNA sequencing technique. Only one C.striaum strain from the same patient was selected in this study, while the repeated ones were excluded.

\section{Antibiotics susceptibility test}

The antibiotic susceptibility test was performed using broth microdilution method, and the antibiotics tested include ceftriaxone, imipenem, erythromycin, clindmycin, gentamycin, tetracycline, vancomycin, ciprofloxicin, sulfamethoxazole, rifampin and daptomycin. The operation of susceptibility test and results analysis were performed according to Clinical and Laboratory Standards Institute guidelines (CLSI) [17].

Pulsed-Field Gel Electrophoresis (PFGE)

In general, the whole genomic DNA chromosome of the C. striatum was extracted, and macrorestriction digestion (Swal) and PFGE (CHEF-DR II apparatus; Bio-Rad, Hercules, CA, USA) were performed as previously reported [15]. Macrorestriction patterns were analysed using Dice coefficient with Bionumerics software (Applied Maths, 
Kortrijk, Belgium,Version 5.0). The classification criteria for PFGE analysis was subjectively designated and the clones with similarity index $100 \%$ was classified to be a single type and named with a single capital letter.

\section{In vitro adherence assay}

In vitro adherence abilities on polystyrene surfaces of different C. striatum strains were detected quantitatively in 96-well flat-bottomed microtitre plates with a commonly used method described previously [15]. Generally, aliquots of $200 \mu \mathrm{L}$ of bacterial suspensions [0.2 optical density (OD) at $\lambda=620 \mathrm{~nm}$ ] were added to the microplate wells and incubated for $24 \mathrm{~h}$ at $35^{\circ} \mathrm{C}$. After the suspension content removed, the remaining attached bacteria in each well were fixed with $99 \%$ methanol and stained with $2 \%$ crystal violet. The negative controls contained LB liquid medium only. Then $33 \%$ glacial acetic acid was used to dissolve bound crystal violet and the OD of the solution was determined ( $\lambda=620 \mathrm{~nm}$ ) using an enzyme immunosorbent assay reader (Tecan, Sunrise). The cutoff OD (ODc) was defined as the mean OD of the negative control. The adhesion abilities was classified as nonadherent, weakly, moderately and strongly according to Souza C et al [14].

\section{Mouse lethality assay}

Six different strains with PFGE types and ATCC6940 standard strain were selected to do mouse lethality assay. Generally, overnight cultures of bacteria were washed twice with sterile PBS by centrifugation, and approximately $10^{7}$ CFUs of bacteria for each strain were then added into $200 \mathrm{ml}$ of PBS for inoculation. Eight-week-old BALB/c mice were intraperitoneally injected with the inoculums. The survival rates of mice were monitored for 7 days. Moribund mice were anesthetized and sacrificed prior to death. Mice were housed and monitored daily at the Animal centre of Chinese Center For Disease Control And Prevention, Beijing.

Results

\section{Isolates distribution and Antibiotic resistance feature}

For the 420 isolates analyzed in this study, 329 isolates (78.3\%) were collected from hospital A, 44 isolates (10.5\%) from hospital B and 47 isolates (11.2\%) from hospital C.

Antibiotics susceptibility testing showed that all of the strains were sensitive to vancomycin, linezolid and daptomycin. The total resistance rates to vancomycin, linezoid and daptomycin were all $0.0 \%$, and those to penicillin, cefepime, meropenem, ciprofloxacin, erythromycin, tetracycline, gentamicin, clindamycin, sulfamethoxazole and trimethoprim were 97.6\% (410/420), 97.1\% (408/420), 86.9\% (365/420), 99.0\% (416/420), 97.6\% (410/420), 69.5\% (292/420), 51.4\% (216/420), 97.6\% (410/420) and 95.2\% (400/420), respectively, as shown in Table 1. 
Table 1

Antibiotics susceptibility profiles of 420 C. striatum strains

\begin{tabular}{|c|c|c|c|c|}
\hline \multirow[t]{2}{*}{ Antibiotics } & \multicolumn{3}{|c|}{$M I C(\mu \mathrm{g} / \mathrm{ml})$} & \multirow{2}{*}{$\begin{array}{l}\text { Percentage of resistant isolates,\% } \\
(\mathrm{n} / 420)\end{array}$} \\
\hline & $\mathrm{MIC}_{50}$ & $\mathrm{MIC}_{90}$ & Range & \\
\hline Penicillin & $\geq 8$ & $>64$ & $\leq 1, \geq 4$ & $97.6(410 / 420)$ \\
\hline Cefepime & $\geq 8$ & $>64$ & $\leq 1, \geq 4$ & $97.1(408 / 420)$ \\
\hline Meropenem & $\geq 32$ & $>64$ & $\leq 4, \geq 16$ & $86.9(365 / 420)$ \\
\hline Ciprofloxacin & $\geq 8$ & 64 & $\leq 1, \geq 4$ & $99.0(416 / 420)$ \\
\hline Erythromycin & 32 & 64 & $\leq 0.5, \geq 2$ & $97.6(410 / 420)$ \\
\hline Tetracycline & $\geq 32$ & $>64$ & $\leq 4, \geq 16$ & $69.5(292 / 420)$ \\
\hline Gentamicin & 8 & $\geq 32$ & $\leq 4, \geq 16$ & $51.4(216 / 420)$ \\
\hline Clindamycin & 16 & $>32$ & $\leq 0.5, \geq 4$ & $97.6(410 / 420)$ \\
\hline $\begin{array}{l}\text { Sulfamethoxazole and } \\
\text { trimethoprim }\end{array}$ & $\gtrless_{8 / 152}$ & $\geq_{8 / 152}$ & $\begin{array}{l}\leq 2 / 38, \geq \\
4 / 76\end{array}$ & $95.2(400 / 420)$ \\
\hline Linezolid & $<0.5$ & $<0.5$ & $\leq 2$ & $0(0.0)$ \\
\hline Daptomycin & $<0.5$ & $<0.5$ & $\leq 1$ & $0(0.0)$ \\
\hline Vancomycin & $<0.5$ & $<0.5$ & $\leq 2$ & $0(0.0)$ \\
\hline
\end{tabular}

Based on the susceptibility testing results, $420 \mathrm{C}$. striatum strains can be classified into 19 resistance biotypes, designating as pattern R1-R19. Among these biotypes, four biotypes (R1, R2, R3 and R4) were the dominant biotypes and were resistant or intermediate resistant to most of the antibiotics tested in this study, except vancomycin, linezolid and daptomycin. The resistance features of the 19 resistance biotypes changed diversely, as shown in Table 2. 329 isolates collected from hospital A belonged to 18 resistance biotypes, and the majority of the isolates belonged to R1 (44.4\%,146/329), R2 (23.4\%, 77/329) and R3 (22.5\%, 74/329). 44 isolates collected from hospital B were divided into 10 different resistance biotypes, and 23 isolates (52.3\%), 7 isolates (15.9\%) and 5 isolates (11.4\%) belonged to R1, R2 and R4, respectively. 47 isolates collected from hospital C were classified into 4 resistance biotypes, and 21 isolates (44.7\%), 18 isolates (38.3\%) and 7 isolates (14.9\%) belonged to R1, R3 and $\mathrm{R} 7$, respectively. 
Table 2

Resistance biotypes of 420 C. striatum strains

\begin{tabular}{|c|c|c|c|c|c|c|c|c|c|c|c|c|c|}
\hline \multirow{2}{*}{$\begin{array}{l}\text { Resistance } \\
\text { biotypes }\end{array}$} & \multirow{2}{*}{$\begin{array}{l}\text { No. of } \\
\text { Isolates(n) }\end{array}$} & \multicolumn{12}{|c|}{ Antibiotics } \\
\hline & & VAN & DAP & LNZ & $\mathbf{P}$ & FEP & CIP & SXT & E & CLI & IPM & $\mathrm{TE}$ & GEN \\
\hline R01 & 190 & $S$ & S & S & $\mathrm{R}$ & $\mathrm{R}$ & $\mathrm{R}$ & $\mathrm{R}$ & $\mathrm{R}$ & $\mathrm{R} / \mathrm{I}$ & $\mathrm{R} / \mathrm{I}$ & $\mathrm{R} / \mathrm{I}$ & $\mathrm{R} / \mathrm{I}$ \\
\hline R02 & 85 & $S$ & $S$ & $S$ & $\mathrm{R}$ & $\mathrm{R}$ & $R / I$ & $\mathrm{R}$ & $\mathrm{R}$ & $\mathrm{R} / \mathrm{I}$ & $\mathrm{R} / \mathrm{I}$ & $R / I$ & $S$ \\
\hline R03 & 94 & $S$ & $S$ & $S$ & $\mathrm{R}$ & $\mathrm{R}$ & $\mathrm{R}$ & $\mathrm{R}$ & $\mathrm{R}$ & $\mathrm{R}$ & $R / I$ & S & $R / I$ \\
\hline R04 & 18 & $S$ & $S$ & $S$ & $\mathrm{R}$ & $\mathrm{R}$ & $\mathrm{R}$ & $\mathrm{R}$ & $\mathrm{R}$ & $\mathrm{R}$ & $S$ & $\mathrm{R}$ & $\mathrm{R}$ \\
\hline R05 & 8 & $S$ & $S$ & $S$ & $S$ & $S$ & $\mathrm{R}$ & $S$ & $\mathrm{R} / \mathrm{I}$ & $\mathrm{R} / \mathrm{I}$ & $S$ & $S$ & $S$ \\
\hline R06 & 6 & $S$ & $S$ & $S$ & $\mathrm{R}$ & $\mathrm{R}$ & $\mathrm{R}$ & $\mathrm{R}$ & $\mathrm{R} / \mathrm{I}$ & $\mathrm{R}$ & $\mathrm{R}$ & $S$ & $S$ \\
\hline R07 & 3 & $S$ & S & S & $\mathrm{R}$ & $R / I$ & $\mathrm{R}$ & S & $\mathrm{R}$ & $R / I$ & $\mathrm{R}$ & S & S \\
\hline R08 & 2 & $S$ & $S$ & $S$ & $\mathrm{R}$ & $\mathrm{R}$ & $\mathrm{R}$ & $S$ & $\mathrm{R}$ & $\mathrm{R}$ & $\mathrm{R}$ & $\mathrm{R}$ & I \\
\hline R09 & 2 & $S$ & $S$ & $S$ & $\mathrm{R}$ & $\mathrm{R}$ & $\mathrm{R}$ & $S$ & $\mathrm{R}$ & $\mathrm{R}$ & $\mathrm{R}$ & $\mathrm{R}$ & $S$ \\
\hline R10 & 2 & $S$ & $S$ & $S$ & $\mathrm{R}$ & $\mathrm{R}$ & $\mathrm{R}$ & $\mathrm{R}$ & S & $S$ & $\mathrm{R}$ & $R / I$ & $S$ \\
\hline R11 & 2 & $S$ & S & S & $\mathrm{R}$ & $\mathrm{R}$ & $\mathrm{R}$ & S & $\mathrm{R}$ & $\mathrm{R}$ & $\mathrm{R}$ & S & 1 \\
\hline R12 & 1 & $S$ & $S$ & $S$ & $\mathrm{R}$ & $\mathrm{R}$ & $\mathrm{R}$ & $\mathrm{R}$ & $S$ & $\mathrm{R}$ & $\mathrm{R}$ & $\mathrm{R}$ & $\mathrm{R}$ \\
\hline R13 & 1 & $S$ & $S$ & $S$ & $\mathrm{R}$ & $\mathrm{R}$ & $\mathrm{R}$ & $\mathrm{R}$ & $\mathrm{R}$ & $\mathrm{R}$ & $S$ & $\mathrm{R}$ & $S$ \\
\hline R14 & 1 & $S$ & $S$ & $S$ & $\mathrm{R}$ & $\mathrm{R}$ & $\mathrm{R}$ & $\mathrm{R}$ & $\mathrm{R}$ & $\mathrm{R}$ & $S$ & $S$ & $\mathrm{R}$ \\
\hline R15 & 1 & $S$ & $S$ & $S$ & $\mathrm{R}$ & $S$ & $S$ & $S$ & $\mathrm{R}$ & $\mathrm{R}$ & $S$ & S & $S$ \\
\hline R16 & 1 & $S$ & $S$ & $S$ & $S$ & $S$ & $S$ & $\mathrm{R}$ & $\mathrm{R}$ & $\mathrm{R}$ & $S$ & $S$ & $S$ \\
\hline R17 & 1 & $S$ & $S$ & $S$ & $S$ & $\mathrm{R}$ & $\mathrm{R}$ & $S$ & $S$ & I & $S$ & $S$ & $S$ \\
\hline R18 & 1 & $S$ & $S$ & $S$ & $S$ & $S$ & $S$ & $S$ & $\mathrm{R}$ & $\mathrm{R}$ & $S$ & $S$ & $S$ \\
\hline R19 & 1 & $S$ & $S$ & $S$ & $S$ & $S$ & $S$ & $S$ & $S$ & 1 & $S$ & $S$ & $S$ \\
\hline
\end{tabular}

C. striatum strains were isolated from multiple clinical specimens. The majority of the C. striatum strains were isolated from sputum $(72.1 \%, 303 / 420)$ and BALF specimens $(17.1 \%, 72 / 420)$, and $1.2 \%(5 / 420)$ strains were isolated from blood specimens. Moreover, $52.4 \%$ ( 220/420) specimens were pure cultured with C. striatum, and 47.6\% (200/420) specimens were polymicrobial, as shown in Table 3. 
Table 3

Microbiological characteristics of 420 samples with C. striatum isolation

\begin{tabular}{|ll|}
\hline Parameters & \\
\hline Specimens & Number(\%) \\
\hline Sputum & $303(72.1)$ \\
\hline Bronchoalveolar lavage fluid(BALF) & $72(17.1)$ \\
\hline Whole blood & $5(1.2)$ \\
\hline Hydrothorax and ascites & $6(1.4)$ \\
\hline Cerebrospinal fluid & $2(0.5)$ \\
\hline Urine & $4(1.0)$ \\
\hline Pus & $5(1.2)$ \\
\hline Central venous catheters & $3(0.7)$ \\
\hline Wound secretion & $20(4.8)$ \\
\hline Simultaneously isolated strains from the same specimen, $\mathrm{n}(\%)$ & \\
\hline C.striatum(pure culture) & $220(52.4)$ \\
\hline Klebsiella pneumoniae & $2(0.5)$ \\
\hline Acinetobacter baumannii & $46(11.0)$ \\
\hline Pseudomonas aeruginosa & $63(15.0)$ \\
\hline Methicillin-resistant Staphylococcus aureus(MRSA) & $42(10.0)$ \\
\hline Enterococcus species & $33(7.9)$ \\
\hline Achromobacter xylosoxidans & $5(1.2)$ \\
\hline Candida species & $2(0.5)$ \\
\hline Stenotrophomonas maltophilia & $13(3.1)$ \\
\hline Aspergillus fumigatus & $17(4.0)$ \\
\hline
\end{tabular}

\section{PFGE results}

A total of $107 \mathrm{C}$. striatum strains with different resistance patterns were selected to do PFGE typing and 52 different PFGE types were identified, among which type T36 (R3), type T28 (R1), type T32 (R1) and type T14 (R1) were the dominant clones, accounting for $14.02 \%$ (15/107), 11.21\% (12/107), 5.61\% (6/107), 3.74\% (4/107), respectively. Moreover, the 4 clones presented similar antibiotic susceptibility feature and were all multi-drug resistant. These four clones were mainly distributed in neurosurgery unit and intensive care unit. For T36 clone, it prevailed in hospital A $(40.0 \%, 6 / 15)$ and hospital B $(60.0 \%, 9 / 15)$. T28 clone and T32 clone were only isolated in hospital C (100.0\%,18/18), while T14 clone distributed in hosptial A (50.0\%, 2/4) and hospital C $(50.0 \%, 2 / 4)$ separately. No isolate from hospital B belonged to above four dominant clones. 


\section{In vitro adherence assay}

Totally, 27 C. striatum clinical isolates with different PFGE types were selected to test their in vitro adherence abilities(Table 4). 92.6\% (25/27) isolates could form moderate to strong in vitro adherence abilities on polystyrene surfaces, and the most adhesive isolates were QFS022 (R1,T24), QFS028 (R1,T21), QFS027 (R1,T40), NMFYC225 (R2,T04) and NMFYC339(R1,T42), all of which were multi-drug resistant strains and only sensitive to vancomycin, linezolid and daptomycin. Only two isolates (NMFYC220 [T15] and NMFYC132 [T44]) showed weakly biofilm formation abilities. 
Table 4

In vitro adherence abilities of $28 \mathrm{C}$. Striatum strains with different PFGE types

\begin{tabular}{|c|c|c|c|c|}
\hline Strains & PFGE type & Resistance pattern & $\mathrm{OD}(620 \mathrm{~nm})$ & Adhesion ability \\
\hline ATCC6940 & - & - & 1.114 & +++ \\
\hline QFS 022 & $\mathrm{~T} 24$ & $\mathrm{R} 01$ & 2.044 & +++ \\
\hline QFS 028 & T21 & $\mathrm{R} 01$ & 1.927 & +++ \\
\hline QFS 027 & $\mathrm{~T} 40$ & $\mathrm{R} 01$ & 1.643 & +++ \\
\hline NMFYC 225 & T04 & $\mathrm{R} 02$ & 1.313 & +++ \\
\hline NMFYC339 & T24 & $\mathrm{R} 01$ & 1.129 & +++ \\
\hline NMFYC 338 & $\mathrm{~T} 43$ & $\mathrm{R} 01$ & 1.100 & +++ \\
\hline NMFYC177 & T05 & $\mathrm{R} 01$ & 0.986 & +++ \\
\hline NMFYC 363 & $\mathrm{~T} 47$ & $\mathrm{R} 02$ & 0.952 & +++ \\
\hline NMFYC464 & T49 & $\mathrm{R} 01$ & 0.875 & +++ \\
\hline QFS 026 & T08 & $\mathrm{R} 02$ & 0.865 & +++ \\
\hline NMBM003 & T36 & R03 & 0.809 & +++ \\
\hline NMFYC 236 & $\mathrm{~T} 45$ & $\mathrm{R} 03$ & 0.767 & +++ \\
\hline QFS 015 & T41 & $\mathrm{R} 03$ & 0.675 & ++ \\
\hline QFS024 & $\mathrm{T} 50$ & $\mathrm{R} 01$ & 0.641 & ++ \\
\hline NMBM010 & $\mathrm{T} 28$ & $\mathrm{R} 03$ & 0.618 & ++ \\
\hline NMFYC007 & T32 & $\mathrm{R} 01$ & 0.609 & ++ \\
\hline QFS 007 & T13 & R01 & 0.576 & ++ \\
\hline NMFYC207 & $\mathrm{T} 48$ & $\mathrm{R} 02$ & 0.537 & ++ \\
\hline NMFYC451 & T14 & $\mathrm{R} 01$ & 0.523 & ++ \\
\hline QFS023 & T01 & R15 & 0.480 & ++ \\
\hline NMFYC477 & T38 & $\mathrm{R} 01$ & 0.479 & ++ \\
\hline NMFYC412 & $\mathrm{T} 15$ & $\mathrm{R} 01$ & 0.452 & ++ \\
\hline NMFYC457 & T36 & R01 & 0.450 & ++ \\
\hline NMFYC454 & $\mathrm{T} 40$ & $\mathrm{R} 02$ & 0.424 & ++ \\
\hline NMFYC108 & $\mathrm{T} 45$ & R02 & 0.402 & ++ \\
\hline NMFYC220 & $\mathrm{T} 15$ & $\mathrm{R} 01$ & 0.296 & + \\
\hline NMFYC132 & T44 & R01 & 0.260 & + \\
\hline
\end{tabular}




\section{Mouse lethality assay}

After intraperitoneal injection C. striatum ( $10^{7}$ CFUs), mouses died from day 1 to day 4 . As shown in Table 5, seven strains showed different mouse lethality. The non-dominant clone (Strain NMGYC339, T24) showed the strongest mouse virulence (90\% lethality) and strong in vitro adherence ability, which was isolated from wound secretion. No death was observed in the groups of strain NMFYC177 and strain NMFYC477. 
Table 5

Mouse lethality of 7 different C. striatum strains

\begin{tabular}{|c|c|c|c|c|c|c|c|c|}
\hline \multirow[t]{2}{*}{ Strains } & \multirow[t]{2}{*}{ No. of mouses } & \multicolumn{6}{|c|}{ Date of Intraperitoneal infection with C. Striatum } & \multirow[t]{2}{*}{ Death rate(\%) } \\
\hline & & Day 0 & Day 1 & Day 2 & Day 3 & Day 4 & Day 5-7 & \\
\hline \multirow[t]{4}{*}{ ATCC6940 } & 1 & A & A & $\mathrm{D}$ & - & - & - & \multirow[t]{4}{*}{$40 \%$} \\
\hline & 2 & A & A & A & $\mathrm{D}$ & - & - & \\
\hline & 6 & A & $\mathrm{D}$ & - & - & - & - & \\
\hline & 7 & $A$ & $A$ & $\mathrm{D}$ & - & - & - & \\
\hline \multirow[t]{4}{*}{ NMGYC220 } & 1 & A & $\mathrm{D}$ & - & - & - & - & \multirow[t]{4}{*}{$40 \%$} \\
\hline & 4 & $A$ & $\mathrm{D}$ & - & - & - & - & \\
\hline & 7 & $A$ & $A$ & $D$ & - & - & - & \\
\hline & 9 & $A$ & $A$ & $D$ & - & - & - & \\
\hline \multirow[t]{9}{*}{ NMGYC339 } & 1 & $A$ & $A$ & $D$ & - & - & - & \multirow[t]{9}{*}{$90 \%$} \\
\hline & 2 & $A$ & $A$ & $D$ & - & - & - & \\
\hline & 3 & $A$ & $A$ & $A$ & $\mathrm{D}$ & - & - & \\
\hline & 4 & $A$ & $D$ & - & - & - & - & \\
\hline & 5 & $A$ & $A$ & $D$ & - & - & - & \\
\hline & 6 & $A$ & $A$ & $D$ & - & - & - & \\
\hline & 7 & $A$ & $A$ & $\mathrm{D}$ & - & - & - & \\
\hline & 8 & $A$ & $A$ & $D$ & - & - & - & \\
\hline & 9 & $A$ & $A$ & $A$ & $\mathrm{D}$ & - & - & \\
\hline \multirow[t]{4}{*}{ QFS023 } & 3 & $A$ & $A$ & $A$ & $\mathrm{D}$ & - & - & \multirow[t]{4}{*}{$40 \%$} \\
\hline & 7 & $A$ & $\mathrm{D}$ & - & - & - & - & \\
\hline & 8 & $A$ & $A$ & $A$ & $D$ & - & - & \\
\hline & 10 & $A$ & $A$ & $A$ & $D$ & - & - & \\
\hline NMFYC177 & $1-10$ & $A$ & $A$ & $A$ & $A$ & $A$ & $A$ & $0 \%$ \\
\hline NMFYC477 & $1-10$ & $A$ & $A$ & $A$ & $A$ & $A$ & $A$ & $0 \%$ \\
\hline \multirow[t]{3}{*}{ NMFYC457 } & 1 & $A$ & $\mathrm{D}$ & - & - & - & - & \multirow[t]{3}{*}{$30 \%$} \\
\hline & 4 & $A$ & $A$ & $A$ & $A$ & $\mathrm{D}$ & - & \\
\hline & 10 & $A$ & $A$ & $A$ & $\mathrm{D}$ & - & - & \\
\hline
\end{tabular}




\section{Discussion}

As a potential pathogen for hospitalized patients in recent years, C. striatum was found to be isolated from almost all types of clinical samples [18-20], especially for sputum, whole blood, cerespinal fluid. In this study, the 420 C. Striatum were mainly isolated from lower respiratory tract $(89.2 \%, 375 / 420)$, and $52.4 \%$ samples (220/420) were purely cultured with C. striatum. Five C. striatum strains were isolated from whole blood samples, and one of the strains were repeatedly isolated from a immunosuppressed patient with infective endocarditis. Some common multi-drug resistant bacteria were simultaneously isolated with $\mathrm{C}$. striatum in the same sample (53.1\%, 223/420), including Acinetobacter baumannii, Methicillin-Resistant Staphylococcus Aureus (MRSA), ESBLs or carbapenemase producing Klebsiella pneumoniae. This study highlights the higher prevalence of C. striatum from the long stay patients, especially for the ones with specific risk factors $[14,15,21]$.

Consistent with the majority of the investigations previously published[1-3], the C. striatum strains detected in this study were all sensitive to vancomycin and linezolid, but no daptomycin resistant isolate was observed. The resistance rates of $420 \mathrm{C}$. striatum strains to penicillin, cefepime, ciprofloxacin, erythromycin, sulfamethoxazole and trimethoprim and clindamycin were all higher than 95\%. Moreover, it is reported that the usage of parenteral antimicrobial drugs could promote $\mathrm{C}$. striatum acquisition [22] and the resistance rate of $\mathrm{C}$. striatum strains to meropenem reached up to $86.9 \%(365 / 420)$ in this study, which suggested that prescription of carbapenems or other non-sensitive antibiotics should be carefully considered, especially for the patients with risk factors for C.striatum acquisition or infection.

Based on the resistance biotypes of $\mathrm{C}$. striatum to 12 types of antibiotics tested in this study, three dominant resistance biotypes were discriminated, which were all multi-drug resistant. To further analyze the potential differences of resistance patterns of C. striatum from different hospitals, 420 isolates from 3 different hospitals in China were enrolled and analyzed. The isolates from hospital A and hospital $\mathrm{C}$ were separately distributed into at least 10 different resistance biotypes, while the isolates from hospital B were intensively distributed in 4 biotypes. The resistance features of the isolates from different hospitals varied greatly based on the results of this study. However, owing to relatively few isolates from hospital B and hospital C, the actual differences of resistance patterns of $\mathrm{C}$. striatum isolates among these 3 hospitals still need to be explored.

To further investigate whether some dominant clones prevailed in the same hospital, PFGE typing method was employed, which revealed that 2 dominant clones (T36 and T14) were isolated from different patients in hospital A and hospital C and lasted from 2016 to 2019, which suggested that some dominant clones could exist in hospitals for a long time. Consistent with our findings, Baio PV et al [23] reported that dominant C. striatum clones can rapidly spread among inpatients and can exist for a long period in the same hospital [2]. Also, it is revealed that $\mathrm{C}$. striatum could transmit from person-to-persion via the hands of healthcare personel or medical environment [24-26]. Unfortunately, based on the current data we obtained, it is difficult to confirm whether these two clones originated from the same patient in these two hospitals at a long distance. For the remaining two dominant clones (T32 and T28), they were only isolated from hospital $\mathrm{C}$ and the two times of nosocomial outbreak were observed from April, 2019 to May, 2019 and both of the outbreaks occured in intensive care unit (ICU). For specific wards such as ICU, more efficient infection control measures should be implemented to better control the transmission of C. striatum.

Souza C et al [14] reported that C. striatum clinical strains can tightly adhere to several kinds of environmental surfaces or implanted medical devices [27-29] and lead to several kinds of invasive infections. Consistently, 
most of the C. striatum strains tested in this study showed moderate to strong biofilm abilities and differed greatly. It is also noteworthy that all of the 4 dominant PFGE clones showed moderate adherence abilities on polystyrene surface, while the most adhesive strains belonged to non-dominant clones and the top three strains were all collected from hospital B. More attention should be taken for preventing catheter-related infections by $\mathrm{C}$. striatum, especially for the strains with strong adherence abilities. Furthermore, as far as we know, it is the first study to reveal the significant mouse lethality of C. striatum. Also, the consistency between in vitro adherence and in vivo mouse lethality of the $\mathrm{C}$. striatum strains was not observed in this study. The actual pathogenicity of C. striatum strains and possible mechanism deserves to be further investigated.

\section{Conclusions}

Most of the C. striatum clinical strains analyzed in this study showed multi-drug resistant features and could exist in hospitals for a long period. The antibiotic resistance patterns of $\mathrm{C}$. striatum strains differed greatly from different districts, and most of the $\mathrm{C}$. striatum strains can present with moderate to strong in vitro adherence abilities on polystyrene surfaces and some clones could lead to significant mouse lethality. The pathogenicity mechanism of multi-drug resistant C.striatum should be further explored to better prevent and control acquisition or infection of C. striatum.

\section{Declarations}

\section{Acknowledgements}

We would like to thank the National Institute for Communicable Disease Control and Prevention and Animal centre of Chinese Center For Disease Control And Prevention for their help in faciliatating this study.

\section{Ethics approval and consent to participate}

The animal study was approved by the Laboratory Animal Welfare\&Ethics Committee of Chinese Center For Disease Control And Prevention (Animal ethics approval no. 2018018).

\section{Consent for publication}

Not applicable.

\section{Availability of data and material}

Not applicable.

\section{Competing interests}

None

\section{Funding}

This study is supported by Major project of Affiliated hospital of Inner Mongolia Medical University (NYFY ZD 012), Program for Young Talents of Science and Technology in Universities of Inner Mongolia Autonomous Region (No. NJYT-17-B06), and National natural science foundation (No. 81660352). 


\section{Authors' contributions}

Xiaohong Shi, Jian Zhang and Yanqiu Han are responsible for the isolates isolation and identification.Yingying LV and Roushan Liu are responsible for in vitro susceptibility test and in vitro adherence abilities. Xiaoli Du and Yingying LV are responsible for PFGE typing experiment. Xuancheng Lu and Yuan Chai are responsible for animal lethality assay. Junrui Wang is responsible for experiment design, data analysis and manuscript writing.

\section{References}

1. Suh JW, Ju Y, Lee CK, Sohn JW, Kim MJ, Yoon YK. Molecular epidemiology and clinical significance of Corynebacterium striatum isolated from clinical specimens. Infect Drug Resist. 2019; 12: 161-171.

2. Wang X, Zhou H, Chen D, Du P, Lan R, Qiu X, et al. Whole-Genome Sequencing Reveals a Prolonged and Persistent Intrahospital Transmission of Corynebacterium striatum, an Emerging Multidrug-Resistant Pathogen. J Clin Microbiol. 2019; 57. pii: e00683-19.

3. Neemuchwala A, Soares D, Ravirajan V, Marchand-Austin A, Kus JV, Patel SN. In Vitro Antibiotic Susceptibility Pattern of Non-diphtheriae Corynebacterium Isolates in Ontario, Canada, from 2011 to 2016. Antimicrob Agents Chemother. 2018; 62(4). pii: e01776-17

4. Hagiya H, Kimura K, Okuno H, Hamaguchi S, Morii D, Yoshida H,et al. Bacteremia due to high-level daptomycinresistant Corynebacterium striatum: A case report with genetic investigation. J Infect Chemother. 2019; 25: 906908.

5. Ajmal S, Saleh OA, Beam E.Development of High-Grade Daptomycin Resistance in a Patient Being Treated for Corynebacterium striatum Infection. Antimicrob Agents Chemother. 2017 ; 61(7). pii: e00705-17.

6. Goldner NK, Bulow C, Cho K, Wallace M, Hsu FF, Patti GJ, et al. Mechanism of High-Level Daptomycin Resistance in Corynebacterium striatum. mSphere. 2018; 3(4). pii: e00371-18.

7. Ramos JN, Souza C, Faria YV, da Silva EC, Veras JFC, Baio PVP, et al. Bloodstream and catheter-related infections due to different clones of multidrug-resistant and biofilm producer Corynebacterium striatum. BMC Infect Dis. 2019; 19:672.

8. Naqvi SY, Salama IG, Narins C, Stuver T. Corynebacterium striatum prosthetic valve endocarditis with severe aortic regurgitation successfully treated with transcatheter aortic valve replacement. BMJ Case Rep. 2018; 11(1). pii: e226881.

9. Roy M, Ahmad S.Rare case of Corynebacterium striatum septic arthritis. BMJ Case Rep. 2016 ; 2016 . pii: bcr2016216914.

10. Wang J, Du X, Cui J, Guo S, Fu Q, Wang Y, et al. Drug susceptibility and homologous analysis on Corynebacterium striatum strains isolated from inpatients. Zhonghua Yi Xue Za Zhi . 2014; 94: $2501-5$. 11. Kang SJ, Choi SM, Choi JA, Choi JU, Oh TH, Kim SE, et al. Factors affecting the clinical relevance of Corynebacterium striatum isolated from blood cultures. PLoS One. 2018; 13: e0199454.

12. Iaria C, Stassi G, Costa GB, Biondo C, Gerace E, Noto A, et al. Outbreak of multi-resistant Corynebacterium striatum infection in an Italian general intensive care unit. J Hosp Infect. 2007; 67:102-4

13. Renom F, Garau M, Rubi M, Ramis F, Galmes A, Soriano JB. Nosocomial outbreak of Corynebacterium striatum infection in patients with chronic obstructive pulmonary disease. J Clin Microbiol. 2007; 45: 2064-7. 14. Souza Cd, Faria YV, Sant'Anna Lde O, Viana VG, Seabra SH, Souza MC, et al. Biofilm production by multiresistant Corynebacterium striatum associated with nosocomial outbreak. Mem Inst Oswaldo Cruz. 2015; 110: $242-8$.

15. Wang J, Wang Y, Du X, Cui J, Wang K, Zhang L, et al. Rapid transmission of multidrug-resistant 
Corynebacterium striatum among susceptible patients in a tertiary hospital in China. J Infect Dev Ctries. 2016; 10: 1299-1305

16. Souza C, Simpson-Louredo L, Mota HF, Faria YV, Cabral FO, Colodette SDS, et al. Virulence potential of Corynebacterium striatum towards Caenorhabditis elegans. Antonie Van Leeuwenhoek. 2019; 112: $1331-1340$.

17. Clinical and Laboratory Standards Institute. Methods for antimicrobial dilution and disk susceptibility testing of infrequently isolated or fastidious bacteria. CLSI document M45-2A. Wayne, PA: CLSI, 2010.

18. Qin L, Sakai Y, Bao R, Xie H, Masunaga K, Miura M, et al. Characteristics of Multidrug-resistant Corynebacterium spp. Isolated from Blood Cultures from Hospitalized Patients in Japan. Jpn J Infect Dis. 2017; 70:152-157.

19. McMullen AR, Anderson N, Wallace MA, Shupe A, Burnham CA. When Good Bugs Go Bad: Epidemiology and Antimicrobial Resistance Profiles of Corynebacterium striatum, an Emerging Multidrug-Resistant, Opportunistic Pathogen. Antimicrob Agents Chemother. 2017; 61. pii: e01111-17.

20. Verroken A, Bauraing C, Deplano A, Bogaerts P, Huang D, Wauters G, et al. Epidemiological investigation of a nosocomial outbreak of multidrug-resistant Corynebacterium striatum at one Belgian university hospital. Clin Microbiol Infect. 2014; 20: 44-50.

21. Otsuka Y, Ohkusu K, Kawamura Y, Baba S, Ezaki T, Kimura S. Emergence of multidrugresistant Corynebacterium striatum as a nosocomial pathogen in long-term hospitalized patients with underlying diseases. Diagn Microbiol Infect Dis. 2006; 54:109-114

22. Hahn WO, Werth BJ, Butler-Wu SM, Rakita RM. Multidrug-resistant Corynebacterium striatum associated with increased use of parenteral antimicrobial drugs. Emerg Infect Dis. 2016; 22:1908-1914. 23. Baio PV, Mota HF, Freitas AD, Gomes DL, Ramos JN, Sant'Anna LO, et al. Clonal multidrug-resistant Corynebacterium striatum within a nosocomial environment, Rio de Janeiro, Brazil. Memorias do Instituto Oswaldo Cruz. 2013; 108: 23-9.

24. Leonard RB, Nowowiejski DJ, Warren JJ, Finn DJ, Coyle MB. Molecular evidence of person-to-person transmission of a pigmented strain of Corynebacterium striatum in intensive care units. J Clin Microbiol. 1994; 32: 164-9.

25. Brandenburg AH, van Belkum A, van Pelt C, Bruining HA, Mouton JW, Verbrugh HA. Patient-to-patient spread of a single strain of Corynebacterium striatum causing infections in a surgical intensive care unit.J Clin Microbiol. 1996; 34: 2089-94

26. Renom F, Gomila M, Garau M, Gallegos MD, Guerrero D, Lalucat J, et al. Respiratory infection by Corynebacterium striatum: epidemiological and clinical determinants. New microbes and new infections. 2014; 2:106-14.

27. De Arriba JJ, Blanch JJ, Mateos F, Martinez-Alfaro E, Solera J. Corynebacterium striatum first reported case of prosthetic valve endocarditis. J Infect. 2002; 44(3):193.

28. Yang HS, Kim YJ, Cho SY, Shin E, Lee HJ. Central venous catheter-related bloodstream infection by Corynebacterium striatum identified by $16 \mathrm{~S}$ rRNA and rpoB gene sequencing. Ann Lab Med. 2015; 35(5):548-50 29. Oliva A, Belvisi V, lannetta M, Andreoni C, Mascellino MT, Lichtner M, et al. Pacemaker lead endocarditis due to multidrug-resistant Corynebacterium striatum detected with sonication of the device. J Clin Microbiol. 2010; 48(12):4669-71.

\section{Figures}




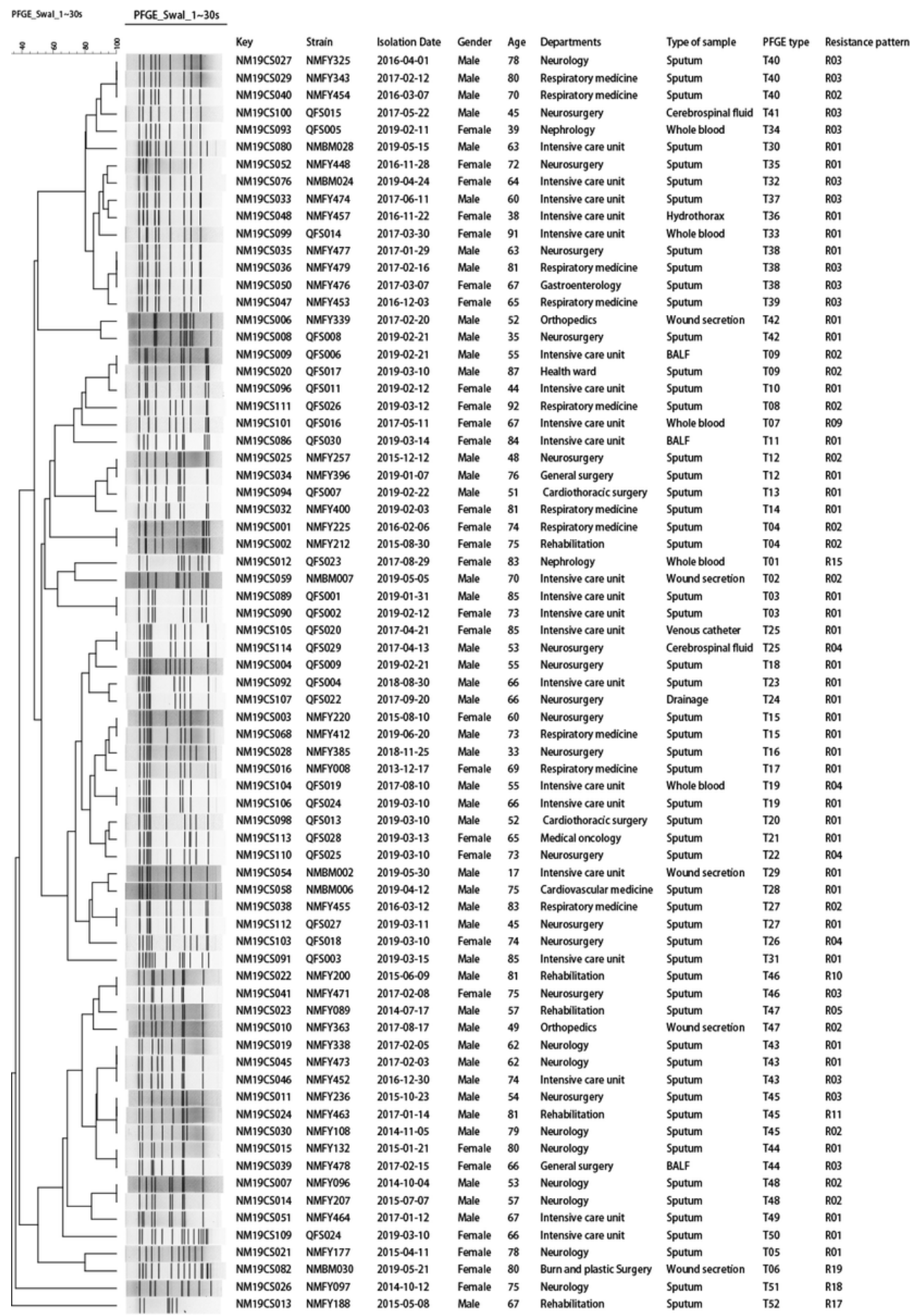

Figure 1 